\title{
Probing Elusive Cations: Infrared Spectroscopy of Protonated Acetic Acid
}

\author{
Julia A. Davies, ${ }^{\text {a) }}$ Nicholas A. Besley, ${ }^{\text {b) }}$ Shengfu Yang ${ }^{\text {a) }}$ and Andrew M. Ellis ${ }^{\mathrm{a},{ }^{*}}$
}

a) Department of Chemistry, University of Leicester, University Road, Leicester, LE1 7RH, UK

b) School of Chemistry, University of Nottingham, University Park, Nottingham, NG7 2RD, UK

*Corresponding author: Email: andrew.ellis@le.ac.uk

Manuscript submitted to The Journal of Physical Chemistry Letters 


\begin{abstract}
Protonated carboxylic acids, $(\mathrm{RCOOH}) \mathrm{H}^{+}$, are the initial intermediates in acid-catalyzed (Fischer) esterification reactions. However the identity of the isomeric form is under debate. Surprisingly, no optical spectra have been reported for any isomer of the protonated carboxylic acid monomer, despite it being a fundamental organic cation. Here, we address these issues by using a new approach to prepare cold He-tagged cations of protonated acetic acid (AA), which entails electron ionization of helium nanodroplets containing metastable dimers of AA. The protonated species is subsequently probed using infrared photodissociation spectroscopy and, following a comparison with calculations, we identify the two isomers whose roles are debated in Fischer esterification. These are the carbonyl-protonated $E, Z$ isomer and the metastable hydroxyl-protonated isomer. Our technique provides a novel approach that can be applied to other elusive ionic species.
\end{abstract}

\title{
TOC Graphic
}

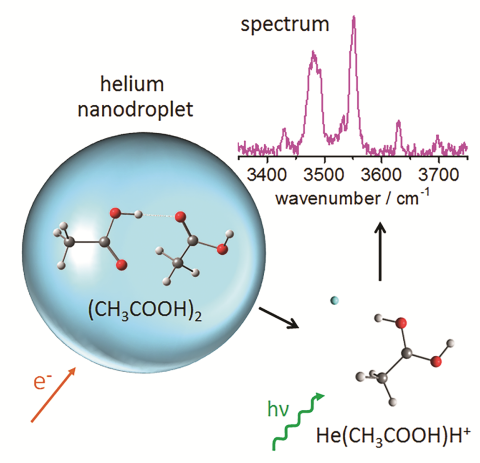


The mechanism of the acid-catalyzed (Fischer) esterification of carboxylic acids was first explored in detail in the 1930s. ${ }^{1,2}$ This early mechanistic work suggested that initial protonation occurs at the hydroxyl oxygen atom. In the case of an acetic acid monomer (AA), this leads to formation of the structure labelled as prot-OH in Figure 1. However, NMR studies of the protonation of simple carboxylic acids carried out in the 1960s indicated that protonation occurs exclusively at the carbonyl oxygen., ${ }^{3,4}$ The NMR work was performed under aggressive conditions with essentially 1:1 concentrations of magic acid $\left(\mathrm{FSO}_{3} \mathrm{H}-\mathrm{SbF}_{5}\right)$ and carboxylic acid. At $-60{ }^{\circ} \mathrm{C}$ a double peak structure was observed, indicating that a single isomer, the $E, Z$ isomer, is formed (Figure 1). It is now a standard assumption in modern organic chemistry textbooks that protonation occurs at the carbonyl oxygen in Fischer esterification. ${ }^{5}$

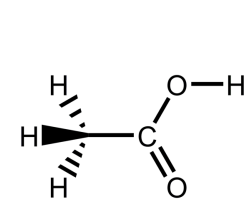

AA

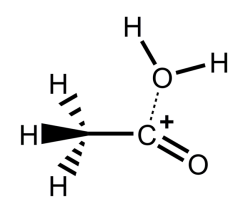

prot-OH

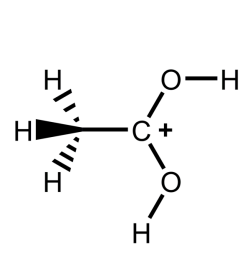

E,Z

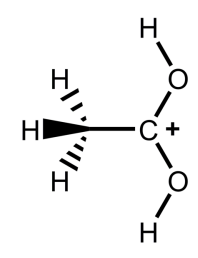

$E, E$

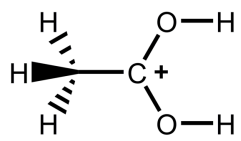

$Z, Z$

Figure 1. Illustration of the molecular structure of neutral acetic acid (AA) and isomers of the protonated species.

However, a recent study combining DFT calculations with electrospray ionization mass spectrometry reached a different conclusion. ${ }^{6}$ This work suggests that carbonyl protonation 
produces an intermediate which is too stable to react with an alcohol to yield an ester. Instead, they found that only hydroxyl protonation can lead to subsequent reaction and this occurs because of the production of the reactive acylium ion ( $c f$. prot-OH structure in Figure 1). This proposal agrees with the original mechanistic predictions from the $1930 \mathrm{~s},{ }^{1,2}$ creating new uncertainty about the reaction mechanism.

The protonated monomer of AA has previously been studied using ab initio calculations and mass spectrometry. ${ }^{7-12}$ However, despite being a rather fundamental organic cation, no optical spectra have been reported for it or for the monomer of other simple protonated carboxylic acids, $(\mathrm{RCOOH}) \mathrm{H}^{+}$. Inokuchi and Nishi have measured infrared (IR) photodissociation spectra in the $\mathrm{OH}$ stretching region for protonated formic acid cluster ions, $(\mathrm{HCOOH})_{n} \mathrm{H}^{+}$, and their AA analogues, for $n \geq 2 \cdot{ }^{13}$ However, this technique could not access the protonated monomers because the IR photon has insufficient energy to induce fragmentation. In a separate study, Hu et al. reported IR photodissociation spectra of protonated AA cluster ions. ${ }^{14}$ However, instead of seeing a reduction in ion signal at the mass of the protonated monomer, $\mathrm{m} / \mathrm{z}$ 61, an enhancement was observed that was attributed to IR absorption by a larger ion, $\left(\mathrm{CH}_{3} \mathrm{COOH}\right) \mathrm{COOH}^{+}$, which subsequently fragmented to give $\left(\mathrm{CH}_{3} \mathrm{COOH}\right) \mathrm{H}^{+}$and $\mathrm{CO}_{2}$. A similar problem was encountered in the case of protonated propanoic acid. ${ }^{15}$

We introduce an experimental technique for measuring IR spectra of He-tagged cations, which utilizes electron ionization of neutral clusters embedded in helium nanodroplets. Mass spectrometry has been used extensively to study electron and multiphoton ionization of doped droplets, ${ }^{16}$ but there have been few IR spectroscopic studies of the ions produced in such processes. ${ }^{17}$ In Figure $2 \mathrm{~b}$ and c, we present IR depletion spectra for He-tagged protonated AA, $\mathrm{He}_{N^{-}}\left(\mathrm{CH}_{3} \mathrm{COOH}\right) \mathrm{H}^{+}$, where $N=1(\mathrm{~m} / z$ 65) and $3(\mathrm{~m} / z$ 73). A spectrum for the untagged ion $(N=$ 
0 at $m / z$ 61) is shown in the Supporting Information (Figure S1) rather than here because it contains broad spectral features due to hot bands, as well as congestion arising from competing photofragmentation pathways. In contrast, spectra for the He-tagged species show relatively narrow and well resolved features.

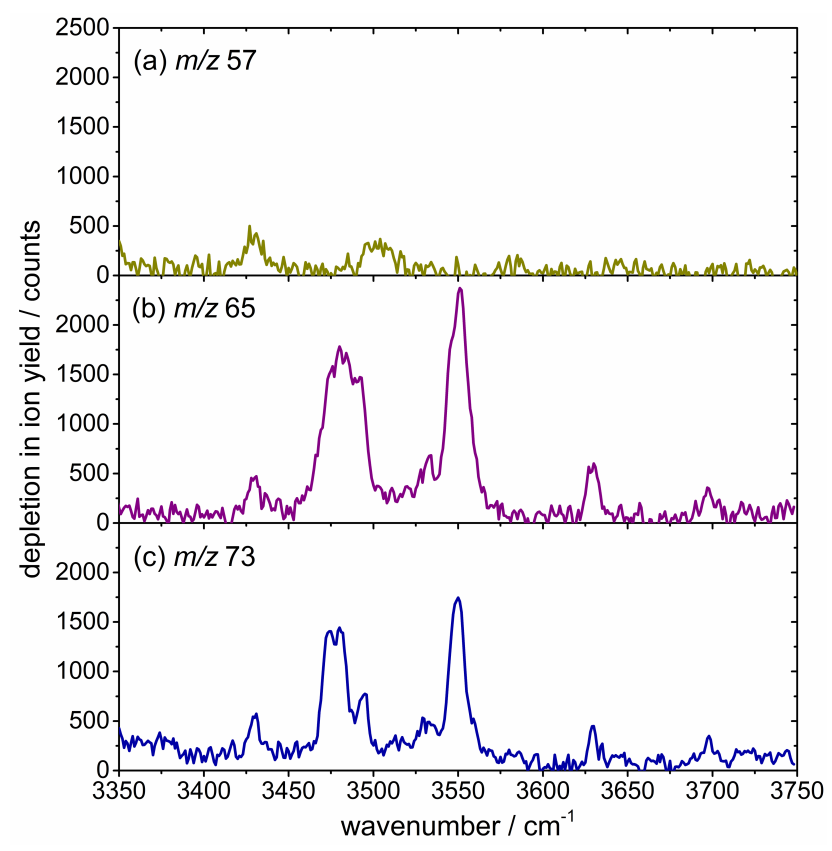

Figure 2. Infrared depletion spectra recorded by monitoring mass channels with $\mathrm{m} / \mathrm{z} 57$, 65 and 73. Those IR bands observed in panels (b) and (c) which are not seen in the lower mass channel in panel (a) are assigned to $\mathrm{OH}$ stretching modes in He-tagged protonated acetic acid.

Also shown in Figure 2 is an IR spectrum recorded at $m / z$ 57. Because this corresponds to a mass of one helium atom below that of an isolated protonated AA monomer, the observed depletion in ion signal cannot be attributed to IR absorption by protonated AA. However it is possible that spectral features from a smaller ion could appear in mass channels 65 and 73 
through the addition of more helium atoms. The spectrum at $\mathrm{m} / \mathrm{z} 57$ therefore acts as a background spectrum and the two weak features at $\sim 3430$ and $\sim 3505 \mathrm{~cm}^{-1}$ are assigned to $\mathrm{He}_{3}-$ $\mathrm{HOCO}^{+} .{ }^{18}$ Consequently, peaks observed at $\sim 3430 \mathrm{~cm}^{-1}$ in the $m / z 65$ and 73 spectra are assigned to $\mathrm{He}_{5}-\mathrm{HOCO}^{+}$and $\mathrm{He}_{7}-\mathrm{HOCO}^{+}$, respectively.

We now turn our attention to the new features observed at $m / z 65$ and 73 , which are not present at $\mathrm{m} / \mathrm{z} 57$, and are therefore assignable to IR absorption by He-tagged protonated AA. Most notable are the two strongest peaks at $\sim 3480$ and $\sim 3550 \mathrm{~cm}^{-1}$. The shape of the lower frequency band clearly changes with the number of attached helium atoms (from $N=1$ to 3 ) and this is attributed to frequency shifts caused by helium solvation effects. Band shifts induced by helium solvation have also been reported for a different cation, $\mathrm{C}_{60}{ }^{+}$, although that study used electronic rather than vibrational spectroscopy. ${ }^{19}$ However, the $N$-dependent behavior is a separate topic and will be the subject of future work. From here onwards, we choose to focus on identifying the structural isomers of protonated AA that are formed.

To assist with the spectral assignments, we have performed ab initio calculations at the MP2/aug-cc-pVTZ level of theory for the four isomers of protonated AA illustrated in Figure 1. For each isomer considered, the ZPE-corrected relative energy, as well as the harmonic vibrational frequencies and intensities, were calculated. We summarize the key findings in Table 1 and present further results in Figure S2 and Table S1. To account for anharmonicity, the calculated $\mathrm{OH}$ stretching frequencies have been scaled by an empirical factor of 0.955 , derived from a comparison of the computed $\mathrm{OH}$ stretching vibrational frequency for the AA neutral monomer and the known experimental gas phase value of $3583 \mathrm{~cm}^{-1} \cdot{ }^{20}$ 
Table 1. Selected results from quantum chemical calculations (MP2/aug-cc-pVTZ) for four isomers of protonated acetic acid.

\begin{tabular}{cccc}
\hline Isomer & Energy $^{\mathrm{a}} / \mathrm{kJ} \mathrm{mol}^{-1}$ & \multicolumn{2}{c}{$\mathrm{OH}_{\text {stretches }} \mathrm{b} / \mathrm{cm}^{-1}$} \\
\hline$E, Z$ & 0.0 & $3470(289)$ & $3539(252)$ \\
$E, E$ & 11.8 & $3548(528)$ & $3558(13)$ \\
$Z, Z$ & 17.0 & $3508(73)$ & $3524(374)$ \\
prot-OH & 28.0 & $3611(66)$ & $3710(138)$ \\
\hline
\end{tabular}

${ }^{a}$ Relative energies including a ZPE correction, plus BSSE for prot-OH.

${ }^{\mathrm{b}}$ Scaled $(\times 0.955)$ harmonic vibrational frequencies with IR intensities given in parentheses in units of $\mathrm{km} \mathrm{mol}^{-1}$.

Before comparing our calculated spectra for the untagged isomers of protonated AA ( $N=$ 0 ) with the experimental spectrum measured at $m / z 65(N=1)$, we consider whether the helium atom causes a significant shift in the $\mathrm{OH}$ stretching frequency. For this purpose, we performed calculations for each isomer with an attached helium atom in various locations relative to the ion, which led to the identification of 18 stable structures (Figures S3 and S4, Table S2). The addition of a single helium atom is found to shift the $\mathrm{OH}$ stretching frequencies by $<2 \mathrm{~cm}^{-1}$ in all but one case: the exception was a He-tagged $Z, Z$ structure, where a shift of $5 \mathrm{~cm}^{-1}$ was calculated. These small shifts allow us to simply compare the four predicted spectra for the bare ions with the Hetagged spectrum recorded here.

In Figure 3, the calculated IR spectra for the untagged isomers are plotted below the spectrum measured at $m / z 65$ for $\mathrm{He}-\left(\mathrm{CH}_{3} \mathrm{COOH}\right) \mathrm{H}^{+}$. Initially, we focus on the assignment of the two strong IR bands centered at 3482 and $3550 \mathrm{~cm}^{-1}$ in the experiments. Comparison with the 
computed spectra indicates that the $E, E, E, Z$ and $Z, Z$ conformers have calculated IR fundamental bands in this region. However, conformers $E, E$ and $Z, Z$ each have one strong and one weak absorption band whereas $E, Z$ has two bands of approximately equal intensity. The pair of experimental IR features also have similar intensities and therefore an assignment to conformer $E, Z$ is considered first. We find good agreement, to within $12 \mathrm{~cm}^{-1}$, in the experimental and calculated vibrational frequencies for both bands and, in addition, the calculated separation between the two bands $\left(69 \mathrm{~cm}^{-1}\right)$ is almost identical to the experimental separation $\left(68 \mathrm{~cm}^{-1}\right)$, which provides strong support for an assignment to the $E, Z$ isomer.
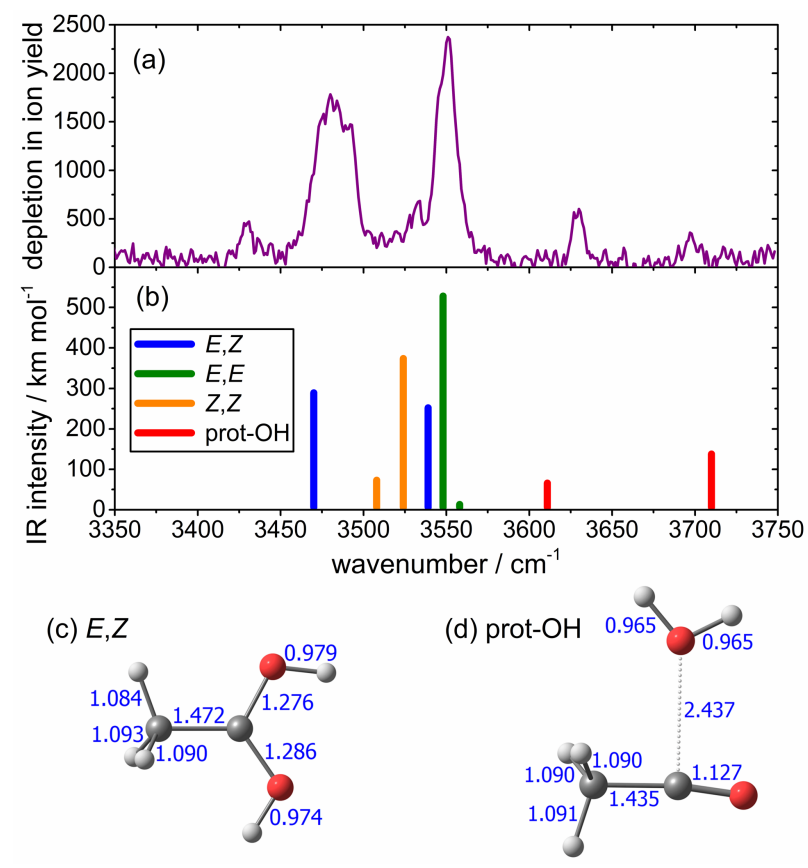

Figure 3. (a) Experimental infrared depletion spectrum recorded by detecting ions at $\mathrm{m} / \mathrm{z} 65$, compared with (b) calculated spectra for four isomers of protonated acetic acid (MP2/aug-ccpVTZ). The calculated vibrational frequencies have been scaled by a factor of 0.955 to account for anharmonicity. Calculated structures are shown for (c) the lowest energy isomer $(E, Z)$ and (d) the metastable isomer prot-OH. The distances shown are in $\AA$. 
Next we consider whether the $Z, Z$ and $E, E$ conformers could be responsible for any of the experimental features in Figure 3a. The weak band at $3533 \mathrm{~cm}^{-1}$ could potentially be assigned to the $Z, Z$ conformer, or alternatively to a combination band of the $E, Z$ conformer that gains intensity via coupling to the strong $E, Z$ band at $3550 \mathrm{~cm}^{-1}$. Furthermore, the $E, E$ conformer could provide a minor contribution to the intensity of the $3550 \mathrm{~cm}^{-1}$ band, but again the evidence is circumstantial. In summary, the formation of isomers $E, E$ and $Z, Z$ can't be ruled out, but if they are present then the populations are likely to be small and therefore we will not discuss them further.

We now turn to the two weak, higher frequency bands centered at 3629 and $3698 \mathrm{~cm}^{-1}$ (Figure 3a). These features are absent from the spectrum at $m / z 57$ (Figure 2a) and are therefore assigned to He-tagged protonated AA. However, we can immediately exclude the $\mathrm{OH}$ stretching fundamentals for isomers $E, E, E, Z$ and $Z, Z$ because the calculated frequencies are at least $70 \mathrm{~cm}^{-1}$ to the red of both experimental bands (Figure 3). We have also considered assignments to overtone and combination bands for the $E, Z$ conformer, both for the bare and He-tagged ions. For this purpose, anharmonic vibrational frequency calculations were performed. The calculated IR intensities for all overtone and combination bands between 3300 and $4000 \mathrm{~cm}^{-1}$ were found to be $<5 \mathrm{~km} \mathrm{~mol}^{-1}$, which is very weak compared with the $\mathrm{OH}$ stretching fundamental transitions of the $E, Z$ conformer $\left(>250 \mathrm{~km} \mathrm{~mol}^{-1}\right)$. Further reasons for discounting these bands are provided in the Supporting Information.

A more viable assignment is to the $\mathrm{OH}$ stretching fundamentals of isomer prot- $\mathrm{OH}$, for which the calculated and experimental band frequencies agree to within $18 \mathrm{~cm}^{-1}$ and this close agreement is illustrated in Figure 3. Furthermore, the calculated band intensities of $>65 \mathrm{~km} \mathrm{~mol}^{-1}$ 
(Table 1) are at least an order of magnitude higher than those predicted for overtone and combination bands in this spectral region, which also supports this assignment. The calculated structure for isomer prot-OH (Figure 3d) indicates that hydroxyl protonation results in a substantial lengthening of one of the C-O bond lengths to $2.437 \AA$, such that we are left essentially with the acylium ion, $\mathrm{CH}_{3} \mathrm{CO}^{+}$, forming a weakly bound complex with $\mathrm{H}_{2} \mathrm{O}$. Indeed, the experimental bands centered at 3629 and $3698 \mathrm{~cm}^{-1}$ lie fairly close to the known $\mathrm{OH}$ stretching frequencies for a gas-phase water monomer but are red-shifted, as expected when complexation occurs.

Another facet of these experiments is that they enable the measurement of spectra not only for the He-tagged cation, but also the neutral precursor. Knowledge of the precursor structure can then be used to predict which cation structures are likely to form following electron ionization. Our technique has the further benefit of rapid cooling by the surrounding helium, enabling access to metastable structures. For example, for AA we have previously shown that two metastable dimers of neutral AA are formed inside helium nanodroplets rather than the lowest energy cyclic dimer. ${ }^{21}$ The two dimers are structurally similar but have distinct hydrogen bonding motifs, a feature that can be exploited to generate different protonated AA conformers. The calculated dimer structures are shown in Figures $4 \mathrm{a}$ and $\mathrm{b}$, whilst the superimposed blue boxes indicate potential structures for protonated AA that would require minimal structural rearrangement. Remarkably, the identified structures match those assigned in the current study. 

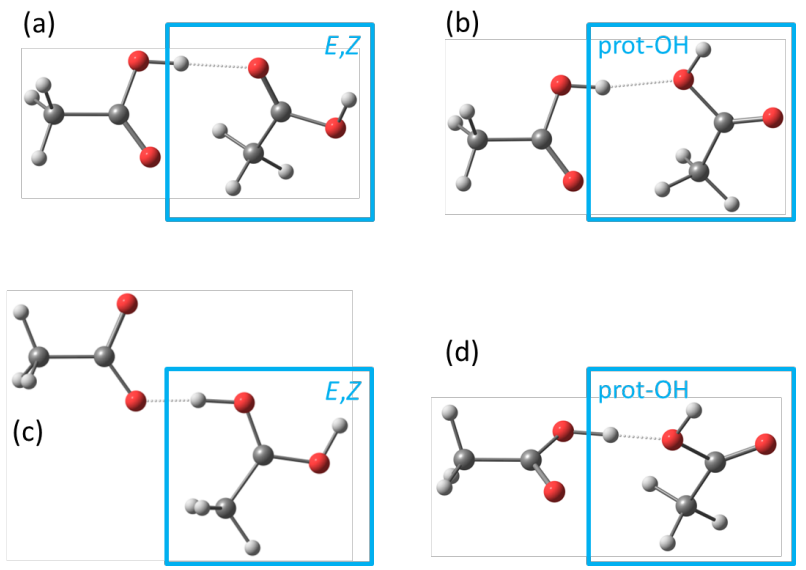

Figure 4. (a) and (b) show the calculated structure of the metastable dimers of neutral acetic acid known to form inside helium nanodroplets. ${ }^{21}$ The blue boxes show entities which closely resemble the protonated acetic acid isomers, $E, Z$ and prot-OH, observed in the current study. Panels (c) and (d) show optimized dimer cation structures following removal of an electron from the neutral dimer structures in (a) and (b), respectively.

Figures $4 \mathrm{c}$ and $\mathrm{d}$ show cation dimer structures that result from geometry optimization of the neutral precursors after removal of an electron, where the latter mimics the experimental ionization process. The blue box in Figure $4 \mathrm{c}$ indicates that energy relaxation leads to formation of the $E, Z$ isomer via a simple proton transfer reaction across a hydrogen bond. A comparison of the structures in $4 \mathrm{~b}$ and $4 \mathrm{~d}$ reveals a $26 \%$ reduction in the calculated distance between donor $\mathrm{H}$ atom and acceptor O-atom, which is progress towards formation of the prot-OH conformer. Therefore, these calculations provide an insight into likely mechanisms for generating the observed protonated AA structures using electron ionization of metastable AA dimers (Figures 4a and $b$ ). Furthermore, the proven capability to create protonated species suggests that this technique has potential applications of biological, as well as chemical, importance. 
Given our assignments, the ability to prepare the $E, Z$ and prot-OH isomers from metastable AA dimers opens the possibility to investigate their potential roles as intermediates in Fischer esterification. ${ }^{6}$ If these isomers were prepared inside helium nanodroplets, just one molecule per droplet, then subsequent pickup and reaction with an alcohol could allow the first stage of the esterification reaction to be studied. Energy to initiate the reaction would be provided by photoabsorption and any subsequent depletion of reactants would be monitored using IR spectroscopy, thus allowing the relative reactivity of the two isomers to be determined. In this way, the helium nanodroplets would act as nanocryostats allowing the isolation and investigation of specific reaction pathways.

In conclusion, we have developed a technique for measuring IR spectra of previously inaccessible cations. To demonstrate its use, we have measured the first IR spectra of the protonated AA monomer, a fundamental organic ion. Comparison with ab initio calculations yields good agreement between theory and experiment. The main experimental features derive from carbonyl protonation and can be attributed to the $E, \mathrm{Z}$ conformer. This is the ionic structure previously reported in NMR experiments ${ }^{3,4}$ and identified in numerous organic chemistry textbooks as the initial intermediate in Fischer esterification. In addition, we have assigned two weak bands to the hydroxyl protonated isomer, a complex of $\mathrm{CH}_{3} \mathrm{CO}^{+}$and $\mathrm{H}_{2} \mathrm{O}$. The possibility exists that this structure, rather than the carbonyl protonated conformer, is the key initial intermediate in Fischer esterification.

\section{Experimental Methods}

Protonated AA was created by electron ionization of liquid helium nanodroplets doped with neutral clusters of AA, a process which generates cations that may be tagged with one or more 
helium atoms. The tagged ions, which are intrinsically cold because weakly bound helium atoms will readily detach from hot ions, were probed by the beam from a tunable IR laser. Spectra were recorded as a function of IR wavelength by monitoring any reduction in ion signal induced by resonant photoabsorption. These measurements were made mass-selectively using a quadrupole mass spectrometer and our technique is a variant of IR photodissociation spectroscopy. The instrument has been described previously ${ }^{21-24}$ but this is the first time it has been used to measure IR spectra for He-tagged ions rather than neutral molecules or clusters inside helium nanodroplets. Further details can be found in the Supporting Information.

\section{Supporting Information}

Experimental details; IR spectra showing fragmentation pathways; computational method; calculated structures, energies and frequencies for bare and He-tagged protonated acetic acid

\section{Acknowledgements}

The authors wish to thank the Leverhulme Trust for providing financial support (grant number RPG-2016-308). This research used the SPECTRE High Performance Computing Facility at the University of Leicester.

\section{References}

1. Ingold, E. H.; Ingold, C. K. Mechanism of the Hydrolysis of Carboxylic Esters with Special Reference to Acid Hydrolysis. J. Chem. Soc. 1932, 0, 758-760.

2. Roberts, I.; Urey, H. C. The Mechanism of Acid Catalyzed Ester Hydrolysis, Esterification and Oxygen Exchange of Carboxylic Acids. J. Am. Chem. Soc. 1939, 61, 2584-2587. 
3. Brookhart, M.; Levy, G. C.; Winstein, S. O-H Chemical Shift, Conformation, and Electron Delocalization in Protonated Carbonyl Compounds. J. Am. Chem. Soc. 1967, 89, 1735-1737.

4. Olah, G. A.; White, A. M. Stable Carbonium Ions. XLIV. The Cleavage of Protonated Aliphatic Carboxylic Acids to Alkyloxocarbonium Ions. J. Am. Chem. Soc. 1967, 89, 35913594.

5. See for example: McMurry, J. Organic Chemistry, $9^{\text {th }}$ edition; Brooks-Cole Publishing: Belmont; 2015.

6. Shi, H.; Wang, Y.; Hua, R. Acid-Catalyzed Carboxylic Acid Esterification and Ester Hydrolysis Mechanism: Acylium Ion as a Sharing Active Intermediate via a Spontaneous Trimolecular Reaction Based on Density Functional Theory Calculation and Supported by Electrospray Ionization-Mass Spectrometry. Phys. Chem. Chem. Phys. 2015, 17, 3027930291.

7. Middlemiss, N. E.; Harrison, A. G. The Structure and Fragmentation of Protonated Carboxylic Acids in the Gas Phase. Can. J. Chem. 1979, 57, 2827-2833.

8. Halim, H.; Schwarz, H.; Terlouw, J. K.; Levsen, K. On the Formation of Protonated Acetic Acid from Ionized n-Alkanoic Acids in the Gas Phase. Org. Mass Spectrom. 1983, 18, 147149.

9. De Koster, C. G.; Terlouw, J. K.; Levsen, K.; Halim, H.; Schwarz, H. On the Formation of $\mathrm{C}_{2} \mathrm{H}_{5} \mathrm{O}_{2}{ }^{+}$Ions Having the Structure of Hydroxyl-Protonated Acetic Acid. Int. J. Mass Spectrom. Ion Proc. 1984, 61, 87-95.

10. Inokuchi, Y.; Nishi, N. Infrared Photodissociation Ppectroscopy of Protonated Formic Acid and Acetic Acid Clusters. J. Phys. Chem. A 2003, 107, 11319-11323. 
11. Miller, C. E.; Francisco, J. S. A Quadratic Configuration Interaction Study of the Proton Affinity of Acetic Acid. Chem. Phys. Lett. 2002, 364, 427-431.

12. Fileti, E. E.; De Oliveeira, A. E.; Morgon, N. H.; Riveros, J. M. Gas-Phase Acylium Ion Transfer Reactions Mediated by a Proton Shuttle Mechanism, Int. J. Quantum Chem. 2011, $111,1596-1606$.

13. Guan, J.; Hu, Y.; Zou, H.; Cao, L.; Liu, F.; Shan, X.; Sheng, L. Competitive Fragmentation Pathways of Acetic Acid Dimer Explored by Synchrotron VUV Photoionization Mass Spectrometry and Electronic Structure Calculations. J. Chem. Phys. 2012, 137, 124308.

14. Hu, Y. J.; Fu, H. B.; Bernstein, E. R. IR plus Vacuum Ultraviolet Spectroscopy of Neutral and Ionic Organic Acid Molecules and Clusters: Acetic Acid, J. Chem. Phys. 2006, 125, 184308.

15. Hu, Y. J.; Fu, H. B.; Bernstein, E. R. IR plus Vacuum Ultraviolet Spectroscopy of Neutral and Ionic Organic Acid Molecules and Clusters: Propanoic Acid, J. Chem. Phys. 2006, 125, 184309.

16. He, Y.; Zhang, J.; Kong, W., Electron Impact Ionization and Multiphoton Ionization of Doped Superfluid Helium Droplets: A Comparison, J. Chem. Phys. 2016, 144, 084302, and references therein.

17. Zhang, X.; Brauer, N. B.; Berden, G.; Rijs, A. M.; Drabbels, M. J. Chem. Phys. 2012, 136, 044305, and references therein.

18. Dopfer, O.; Olkhov, R. V.; Roth, D.; Maier, J. P. Intermolecular Interaction in Proton-Bound Dimers. Infrared Photodissociation Spectra of $\mathrm{Rg}-\mathrm{HOCO}^{+}(\mathrm{Rg}=\mathrm{He}, \mathrm{Ne}, \mathrm{Ar})$ Complexes, Chem . Phys. Lett. 1998, 296, 585-591. 
19. Kuhn, M.; Renzler, M.; Postler, J.; Ralser, S.; Spieler, S.; Simpson, M.; Linnartz, H.; Tielens, A. G. G. M.; Cami, J.; Mauracher, A.; Yang, Y.; Alcami, M.; Martin, F.; Beyer, M. K.; Wester, R.; Lindinger, A.; Scheier, P. Atomically Resolved Phase Transition of Fullerene Cations Solvated in Helium Droplets, Nat. Comm. 2016, 7, 13550.

20. Shimanouchi, T. Tables of Molecular Vibrational Frequencies, Consolidated, Vol. 1, NSRDSNBS 39, National Bureau of Standards, Washington DC, 1972.

21. Davies, J. A.; Hanson-Heine, M. W.; Besley, N. A.; Shirley, A.; Trowers, J.; Yang, S.; Ellis, A. M. Dimers of Acetic Acid in Helium Nanodroplets, Phys. Chem. Chem. Phys. 2019, DOI: 10.1039/C8CP05934A.

22. Tandy, J.; Feng, C.; Boatwright, A.; Sarma, G.; Sadoon, A. M.; Shirley, A.; Rodrigues, N. D. N.; Cunningham, E. M.; Yang, S.; Ellis, A. M. Infrared Spectroscopy of Salt-Water Complexes, J. Chem. Phys. 2016, 144, 121103.

23. Sadoon, A. M.; Sarma, G.; Cunningham, E. M.; Tandy, J.; Hanson-Heine, M. W.; Besley, N. A.; Yang, S.; Ellis, A. M. Infrared Spectroscopy of $\mathrm{NaCl}\left(\mathrm{CH}_{3} \mathrm{OH}\right)_{n}$ Complexes in Helium Nanodroplets, J. Phys. Chem. A 2016, 120, 8085-8092.

24. Sulaiman, M. I.; Yang, S.; Ellis, A. M. Infrared Spectroscopy of Methanol and Methanol/Water Clusters in Helium Nanodroplets: The OH Stretching Region, J. Phys. Chem. A 2017, 121, 771-776. 